\title{
Erratum
}

\section{Infrared Laser Stark Spectroscopy of Ammonia}

K. Shimoda and Y. Ueda

Department of Physics, University of Tokyo, Bunkyo-ku, Tokyo 113, Japan

J. Iwahori

Department of Information Science, Fukui University, Bunkyo, Fukui 910, Japan

Appl. Phys. 21, 181-189 (1980)

The value of $A$ in the fourth column in Table 4 (the $v_{2}$ state, asymmetric) should read 9.699895(50) instead of $9.69895(50)$.

The corrected Table 4 is given below.

Table 4. Molecular constants of ${ }^{14} \mathrm{NH}_{2} \mathrm{D}$ determined from 218 observed lines

\begin{tabular}{llllr}
\hline \multicolumn{5}{c}{ Band origin. 886.2633(6) } \\
Inversion & $\begin{array}{l}\text { The ground state } \\
0.405934(10)\end{array}$ & $\begin{array}{l}\text { The } v_{2} \text { state } \\
20.1800(14)\end{array}$ & \\
doubling & \multicolumn{1}{c}{ Symmetric } & Antisymmetric & Symmetric & Antisymmetric \\
& $9.682907(10)$ & $9.679767(10)$ & $9.826890(40)$ & $9.699895(50)$ \\
$\mathrm{A}$ & $6.412417(10)$ & $6.410285(10)$ & $6.409166(90)$ & $6.360897(120)$ \\
$\mathrm{B}$ & $4.690030(11)$ & $4.690794(11)$ & $4.616861(90)$ & $4.648266(120)$ \\
$\mathrm{C}$ & - & - & & $0.1567(37)$ \\
$\mu_{a}$ & $1.45674(26)+0.93(100) \times 10^{-5} p^{2}$ & & $1.2663(17)$ \\
$\mu_{c}$ & $+1.11(14) \times 10^{-4}\left(p_{a}^{2}+p_{b}^{2}-p_{c}^{2}\right)$ & & \\
\hline
\end{tabular}

